\title{
Replacing PAPS: in vitro phase II sulfation of steroids with the liver S9 fraction employing ATP and sodium sulfate
}

Sumudu A. Weththasinghe1, Christopher C. Waller1, Han Ling Fam¹, Bradley J. Stevenson'1, Adam T. Cawley², Malcolm D. McLeod*1

1Research School of Chemistry, Australian National University, Canberra, ACT, 2601, Australia ${ }^{2}$ Australian Racing Forensic Laboratory, Racing NSW, Sydney, NSW, 2000, Australia

${ }^{*}$ Corresponding Author

Associate Professor Malcolm D. McLeod

Research School of Chemistry, Australian National University, Canberra, ACT, 2601, Australia

Tel: +612 6125 3504; Fax: +612 61250755 E-mail: malcolm.mcleod@anu.edu.au 


\begin{abstract}
:
In vitro technologies provide the capacity to study drug metabolism where in vivo studies are precluded due to ethical or financial constraints. The metabolites generated by in vitro studies can assist antidoping laboratories to develop protocols for the detection of novel substances that would otherwise evade routine screening efforts. In addition, professional bodies such as the Association of Official Racing Chemists (AORC) currently permit the use of in vitro derived reference materials for confirmation purposes providing additional impetus for the development of cost effective in vitro metabolism platforms. In this work alternative conditions for in vitro phase II sulfation using human, equine or canine liver S9 fraction were developed, with adenosine triphosphate (ATP) and sodium sulfate in place of the expensive and unstable co-factor 3'-phosphoadenosine-5'-phosphosulfate (PAPS), and employed for the generation of six representative steroidal sulfates. Using these conditions, the equine in vitro phase II metabolism of the synthetic or so-called "designer" steroid furazadrol $\left(\left[1^{\prime}, 2^{\prime}\right]\right.$ isoxazolo[4',5':2,3]-5 $\alpha$-androstan- $17 \beta$-ol) was investigated, with ATP and $\mathrm{Na}_{2} \mathrm{SO}_{4}$ providing comparable metabolism to reactions using PAPS. The major in vitro metabolites of furazadrol matched those observed in a previously reported equine in vivo study. Finally, the equine in vitro phase II metabolism of the synthetic steroid superdrol (methasterone, $17 \beta$-hydroxy-2 $\alpha, 17 \alpha$-dimethyl-5 $\alpha$ androstan-3-one) was performed as a prediction of the in vivo metabolic profile.
\end{abstract}

\title{
Keywords:
}

sulfate ester, sulfation, in vitro metabolism, PAPS, steroid, anti-doping 


\section{Introduction:}

The development of analytical methods to detect the use of illicit substances such as performanceenhancing Anabolic Androgenic Steroids (AAS) requires a detailed knowledge of drug metabolism.1,2,3 For many agents the parent compound is extensively metabolised and cannot be detected, so metabolites must be targeted as markers of drug administration. Traditionally, the metabolic profile of a steroid is established by in vivo studies that involve drug administration to one or more experimental subjects. Alternatively, in vitro technologies based on cultured liver cells or liver extracts provide methods that mitigate many of the ethical concerns regarding human or animal health and safety.4,5 These issues are of particular relevance in the case of synthetic or so-called "designer" steroids since they are often brought to market in a clandestine fashion, and the majority of these compounds do not have available data regarding their purity, safety, or efficacy. ${ }^{6,7}$ Given this, in vitro experiments would offer a safer approach to identifying metabolite markers.

The metabolism of AAS occurs in two complementary phases: phase I metabolism includes chemical modifications of the steroid skeleton such as reduction, oxidation, or hydroxylation, whereas phase II metabolism includes condensation reactions with other small molecules, termed conjugation. Steroid phase II metabolism usually involves conjugation with polar anionic sulfate or glucuronic acid groups to increase aqueous solubility for excretion, and in many cases these phase II conjugates make up the majority of metabolites detected.1,2,3 As a consequence, phase II metabolites can serve as markers for drug detection, and monitoring directly for these metabolites can offer advantages such as reduced sample preparation. ${ }^{8}$ Other advantages can also arise from the study of phase II metabolites. Monitoring of phase II sulfate conjugates can increase detection windows for some analytes, 9,10,11,12,13,14 and has been used to distinguish between the endogenous or exogenously administered steroids. ${ }^{10,15,16,17}$ Further, the formation and subsequent decomposition of phase II sulfate conjugates has also been implicated in epimerisation of $17 \alpha$-alkyl-17 $\beta$-hydroxy steroids, leading to the formation of $17 \beta$-alkyl-17 $\alpha$-hydroxy compounds and other minor but significant metabolites.18,19,20 Given the importance of phase II metabolism, there is a need for in vitro systems that can faithfully replicate in vivo metabolism. This is particularly relevant in equine and canine sports, as current Association of Official Racing Chemist (AORC) criteria allow for the use of in vitro-derived reference materials in confirmatory analysis. ${ }^{21}$

In vitro technologies typically make use of enzymatic products derived from liver tissue, as the liver is the primary organ involved AAS metabolism. Homogenised liver can be centrifuged at 9,000 g for 20 min to isolate a supernatant commonly referred to as the S9 fraction. ${ }^{22}$ This fraction includes disrupted membranes of the endoplasmic reticulum (microsomes) and the soluble components of the cytosol. The S9 fraction can be fractionated further by ultracentrifugation at $100,000 \mathrm{~g}$ to isolate the microsomal fraction (pellet) from the cytosolic fraction (supernatant) and all three preparations are commercially available. From the perspective of AAS metabolism: microsomes are a concentrated source of cytochrome P450, flavin monooxygenase, and uridine 5'-diphospho-glucuronosyltransferase (UGT) 
enzymes; liver cytosol contains aldehyde oxidase and sulfotransferase (SULT) enzymes; and S9 fraction contains all of these components. Cofactors must be added to these liver extracts for in vitro metabolism: glucuronylation by S9 fraction or microsomes with uridine 5'-diphosphoglucuronic acid (UDPGA), or sulfation (sometimes called sulfonation) by S9 fraction or cytosol with 3'-phosphoadenosine-5'phosphosulfate (PAPS). ${ }^{4}$ However, the PAPS cofactor required for sulfation is prohibitively expensive (US $\$ 1,645$ for $25 \mathrm{mg}$ of PAPS, Sigma-Aldrich ${ }^{23}$ ) and chemically unstable (PAPS $\mathrm{t}_{1 / 2}=20 \mathrm{~h}$ at $\mathrm{pH} \mathrm{8.024}$ ), and as a result in vitro studies are typically limited to phase I metabolism.5,25 There have been some reports detailing the study of phase II steroid metabolism using in vitro systems, ${ }^{26,27,28}$ but to date these have not been widely adopted by laboratories.

The limitations associated with PAPS can be overcome by in situ synthesis based on the physiological biosynthetic pathway (Figure 1).24,29-31 An in vitro approach for the preparation of sulfate metabolites has been described by Burkart et al.24 and employs a series of six bacterial enzymes derived from Rhizobium meiloti and Escherichia coli for the in situ generation of PAPS. Using this approach, ATPsulfurylase catalyses the sulfation of ATP to generate adenosine-5'-phosphosulfate (APS). This compound is subsequently phosphorylated by APS-kinase to generate PAPS and adenosine diphosphate (ADP). In animal cells, these two enzymes are expressed as a bifunctional protein molecule named PAPS synthase (PAPSS). ${ }^{32}$ Following PAPS synthesis, a sulfotransferase (SULT) can then catalyse the sulfation of a target molecule hydroxyl group. In addition to the sulfated metabolite, 3'-phosphoadenosine-5'phosphate (PAP) is released and is subsequently dephosphorylated and re-phosphorylated in several steps to afford ATP. Overall this protocol generated a number of sulfate compounds in high yield, but may be difficult to implement due to the requirement for a number of bacterial enzymes, many of which are not be readily available to laboratories. ${ }^{33}$

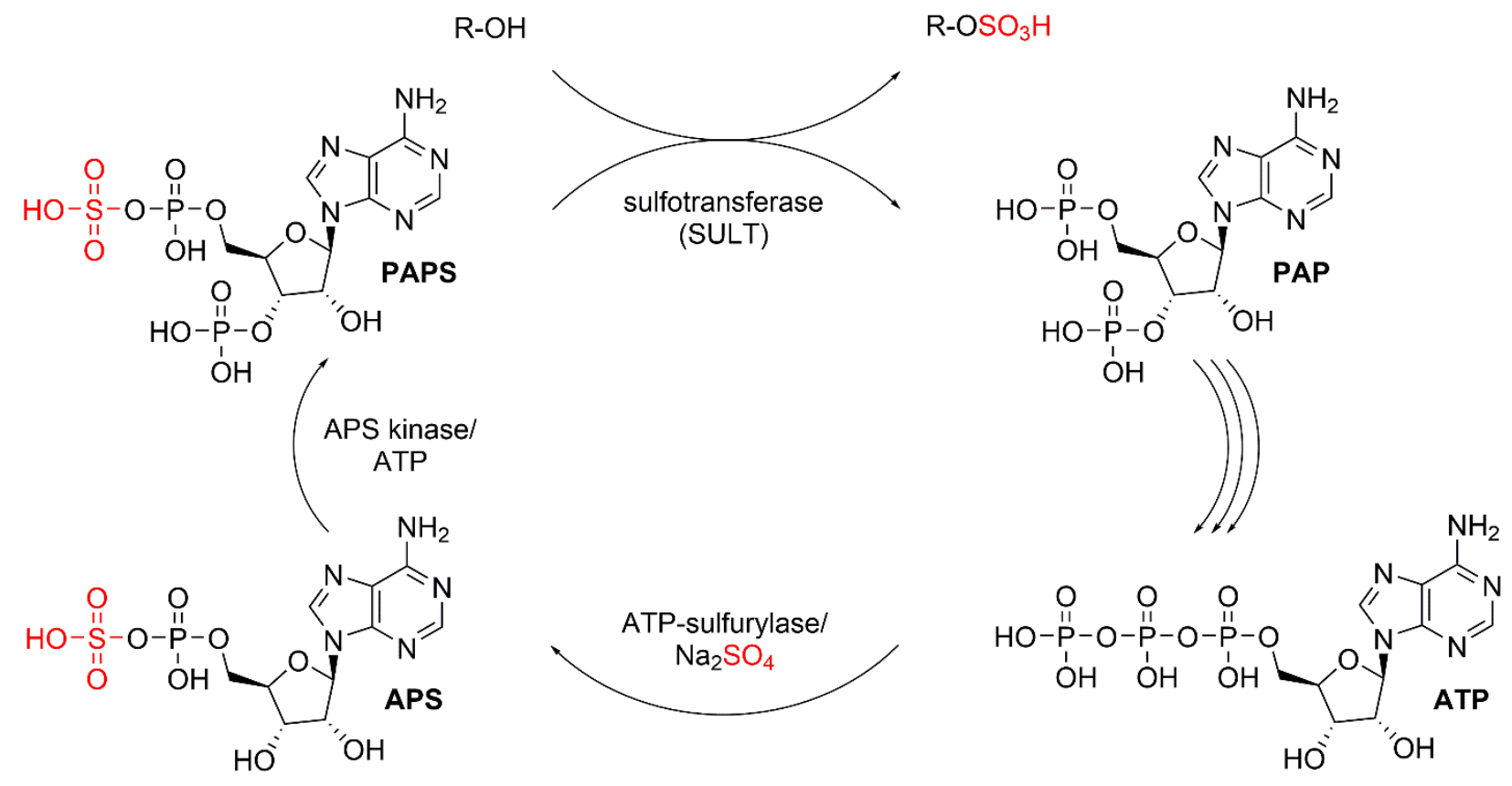


Figure 1: Sulfotransferase promoted sulfation of an acceptor alcohol by PAPS together with the twostep biogenesis of PAPS from ATP and sodium sulfate promoted by ATP sulfurylase and APS kinase. In animal cells, these two enzymes are expressed as a bifunctional protein molecule PAPSS.

Sulfation of xenobiotics is known to occur in the liver, suggesting that the enzymes required for PAPS production would be present in liver preparations. The PAPSS isoform PAPSS $2 \mathrm{~b}$ localises in the cytoplasm and is therefore available for PAPS generation in the liver S9 fraction or cytosol.34,35,36,37 As a result, this study explored the possibility of employing ATP (US $\$ 53$ for $1000 \mathrm{mg}$, Sigma Aldrich ${ }^{38}$ ) and sodium sulfate as inexpensive precursors for the generation of PAPS in situ for in vitro metabolism targeting sulfate metabolites. In particular, the study sought to optimise the experimental conditions and provide a metabolic platform capable of faithfully replicating in vivo sulfation. The optimised conditions have been used to generate equine in vitro phase II metabolic profiles of the synthetic anabolic steroids furazadrol (Figure 2, [1',2']isoxazolo[4',5':2,3]-5 $\alpha$-androstan-17 $\beta$-ol, F) ${ }^{25}$ and superdrol (methasterone, $17 \beta$-hydroxy-2 $\alpha, 17 \alpha$-dimethyl-5 $\alpha$-androstan-3-one, S), ${ }^{5}$ so demonstrating an economical approach for the study of phase II drug metabolism.
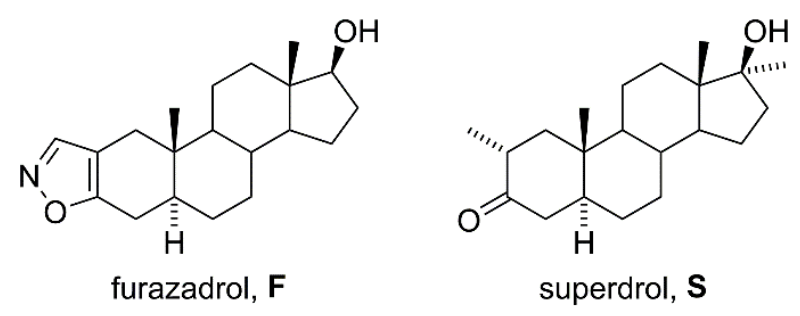

Figure 2: Chemical structures of furazadrol, $\mathbf{F}$ and superdrol, $\mathbf{S}$.

\section{Materials and Methods}

\subsection{Materials}

Chemicals and solvents including lithium tri-sec-butylborohydride (L-Selectride $®$ ) solution in anhydrous tetrahydrofuran (THF), anhydrous $N, N$-dimethylformamide (DMF), magnesium chloride, sulfur-trioxide pyridine complex ( $\mathrm{SO}_{3}$.py), tertiary-butanol $(t$ - $\mathrm{BuOH})$, glucose-6-phosphate (G6P), NADdependant glucose-6-phosphate dehydrogenase (G6PDH) from Leuconostoc mesenteroides, adenosine triphosphate (ATP), 3'-phosphoadenosine-5'-phosphosulfate (PAPS), nicotinamide adenine dinucleotide phosphate (NADP), uridine 5'-diphosphoglucuronic acid (UDPGA), and estrone (3hydroxyestra-1,3,5(10)-trien-17-one, E) were purchased from Sigma-Aldrich (Castle Hill, Australia), and were used as supplied unless otherwise stated. Neutral ATP stock solutions were prepared at $0.1 \mathrm{M}$ with aqueous sodium hydroxide solution. Nicotinamide adenine dinucleotide (NAD) was purchased from Amresco (Solon, USA). Formic acid was purchased from Ajax Chemicals (Auburn, Australia). 1,4Dioxane (dioxane) was purchased from Merck (Darmstadt, Germany). Epiandrosterone (3 $\beta$-hydroxy$5 \alpha$-androstan-17-one, EA), androsterone ( $3 \alpha$-hydroxy- $5 \alpha$-androstan-17-one, $\mathbf{A})$, etiocholanolone $(3 \alpha$ - 
hydroxy-5 $\beta$-androstan-17-one, EC), testosterone (17 $\beta$-hydroxyandrost-4-en-3-one, T) and nandrolone (17ß-hydroxyestra-4-en-3-one, N) were purchased from Steraloids (Newport RI, USA). Superdrol (S) was purchased from the National Measurement Institute (North Ryde, Australia). Epitestosterone (17 $\alpha$ hydroxyandrost-4-en-3-one, ET) was prepared from $\mathbf{T}$ according to literature methods, ${ }^{39}$ Epiandrosterone 3-sulfate (EAS), androsterone 3-sulfate (AS), etiocholanolone 3-sulfate (ECS), estrone 3-sulfate (ES), testosterone 3-sulfate (TS) and nandrolone 17-sulfate (NS) were prepared according to literature methods. ${ }^{40}$ Pooled equine, canine, or human liver S9 fractions were purchased from Sekisui XenoTech (Kansas City, USA). Solid-phase extraction (SPE) was performed using Waters (Rydalmere, Australia) Oasis WAX 6cc cartridges (PN 186004647), or Waters Sep-Pak C18 (3 cc, 500 mg) cartridges (PN WAT020805) as specified. Escherichia coli glucuronylsynthase, and $\alpha$-D-glucuronyl fluoride were prepared according to literature methods. ${ }^{41}$ Escherichia coli NADP-dependant G6PDH was expressed with an N-terminal hexa-histidine tag purified according to previously described methods. ${ }^{42}$ The E. coli BL21 (DE3) with pETMCSIII-g6pdh was kindly provided by Professor David Ollis at the Research School of Chemistry, Australian National University.

\subsubsection{Furazadrol reference materials}

A range of previously synthesised furazadrol reference materials were employed to aid the identification of phase I and phase II metabolites. ${ }^{25,40}$ These were F, isofurazadrol $\left(\left[1^{\prime}, 2^{\prime}\right]\right.$ isoxazolo$\left[4^{\prime}, 3^{\prime}: 2,3\right]-5 \alpha$-androstan-17 $\beta$-ol, IF), epifurazadrol ([1',2']isoxazolo[4', $\left.5^{\prime}: 2,3\right]-5 \alpha$-androstan-17 $\alpha$-ol, EF),

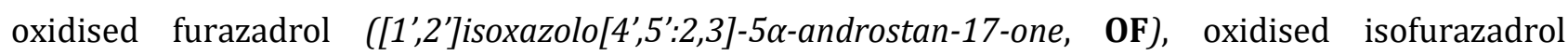
([1',2']isoxazolo[4',3':2,3]-5 $\alpha$-androstan-17-one, OIF), furazadrol 17-sulfate (FS), isofurazadrol 17sulfate (IFS), epifurazadrol 17-sulfate (EFS), furazadrol 17-glucuronide (FG), isofurazadrol 17glucuronide (IFG), and epifurazadrol 17-glucuronide (EFG).

\subsubsection{Superdrol reference materials}

A range of superdrol reference materials were employed to aid the identification of phase I and phase II metabolites. Superdrol (S) was sourced commercially, however the remaining materials were prepared

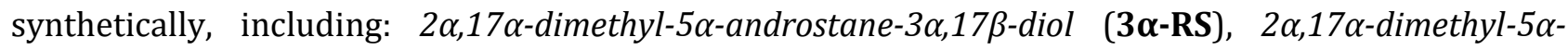

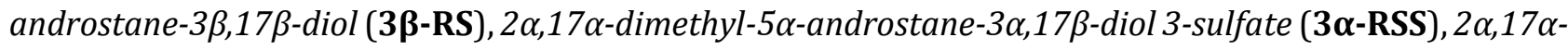

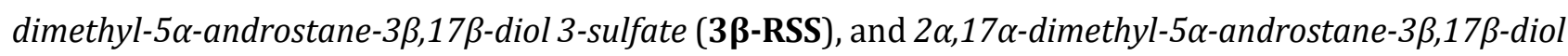
3-glucuronide (3及-RSG). Experimental details, characterisation data, and copies of the ${ }^{1} \mathrm{H}$ NMR, selected ${ }^{13} \mathrm{C}$ NMR, and +EI LRMS or -ESI LRMS spectra are provided in the Supporting Information.

\subsection{Analytical methods}

\subsubsection{LC-MS assay for the optimisation of the in vitro sulfation reaction}

The LC-MS analysis for the optimisation of the in vitro sulfation reaction was performed on an Agilent (Mulgrave Australia) 1260 UHPLC system coupled to an Agilent 6120 quadrupole mass spectrometer equipped with an Agilent Poroshell 120 EC-C18 column $(2.1 \mathrm{~mm}$ x $30 \mathrm{~mm}, 2.7 \mu \mathrm{m})$ incubated at $30{ }^{\circ} \mathrm{C}$ 
and eluting with a gradient consisting of the following mobile phases, A: $10 \%$ methanol in aqueous ammonium acetate solution (10 mM), B: 90\% methanol in aqueous ammonium acetate solution (10 $\mathrm{mM}$ ), gradient: 0-5 min A-B (70:30 v/v) to B (100\%), 5-6 min, B (100\%), 6-9 min B (100\%) to A-B (70:30 $\mathrm{v} / \mathrm{v}$ ), $6 \mathrm{~min}$ re-equilibration, flow rate $0.2 \mathrm{~mL} \mathrm{~min}^{-1}$. Steroid sulfate conjugates were monitored for the anion, [M-H]; using atmospheric pressure electrospray ionization (AP-ESI) and selected ion monitoring. 43

\subsubsection{Furazadrol LC-MS analysis}

The LC-MS analysis for furazadrol metabolites was performed as previously reported. ${ }^{25}$ Positive mode liquid chromatography-high resolution accurate mass (LC-HRAM) spectrometry analysis was undertaken using a Thermo Fisher Scientific (Bremen, Germany) Ultimate 3000 HPLC coupled to an Q Exactive Hybrid Quadrupole Orbitrap mass spectrometer equipped with a Waters SunFire C18 column (100 mm x $2.1 \mathrm{~mm}, 3.5 \mu \mathrm{m}$ ) eluting with a gradient consisting of the following mobile phases, A: $0.1 \%$ formic acid in water, B: 0.1\% formic acid in methanol, gradient: 0-1 min A-B (95:5 v/v), 1-15 min A-B $(95: 5 \mathrm{v} / \mathrm{v})$ to $\mathrm{A}-\mathrm{B}(5: 95 \mathrm{v} / \mathrm{v}), 15-19 \mathrm{~min} \mathrm{~A}-\mathrm{B}(5: 95 \mathrm{v} / \mathrm{v}), 5$ min re-equilibration, flow rate $0.4 \mathrm{~mL} \mathrm{~min}^{-1}$. Unconjugated steroids and steroid glucuronides were monitored for the proton adduct $\left([\mathrm{M}+\mathrm{H}]^{+}\right)$using HESI in positive full scan or targeted MS/MS mode at a resolution of 70,000 (FWHM). Negative mode LC-HRAM spectrometry analysis was undertaken using a Q Exactive Hybrid Quadrupole-Orbitrap mass spectrometer equipped with a Phenomenex (Torrance CA, USA) Gemini C18 column (50 mm x 2 mm, 5 $\mu \mathrm{m})$, eluting with a gradient consisting of the following mobile phases, A: aqueous ammonium acetate (0.01 M, pH 9.0), B: 0.1\% acetic acid in acetonitrile, gradient: 0-2 min A-B (99:1 v/v), 2-8.5 min A-B (99:1 $\mathrm{v} / \mathrm{v}$ ) to $\mathrm{A}-\mathrm{B}(20: 80 \mathrm{v} / \mathrm{v}), 2.7 \mathrm{~min}$ re-equilibration, flow rate $0.5 \mathrm{~mL} \mathrm{~min}^{-1}$. Steroid glucuronide and sulfate conjugates were monitored for the anion ([M-H]-) using HESI in negative full-scan or targeted MS/MS mode at a resolution of 70,000 (FWHM).

\subsubsection{Superdrol LC-MS analysis}

The LC-MS analysis for superdrol metabolites was performed using an Agilent 1290 Infinity II LC system coupled to an Agilent 6545 Q-ToF mass spectrometer equipped with a Phenomenex Gemini C18 column ( $50 \mathrm{~mm} \times 2 \mathrm{~mm}, 5 \mu \mathrm{m}$ ) eluting with the gradient outlined for negative mode LC-MS analysis in section 2.2.2. Steroid glucuronide and sulfate conjugates were monitored for the anion ([M-H]-) using HESI in negative full-scan or targeted MS/MS mode.

\subsection{In vitro phase II metabolism}

\subsubsection{Optimising sulfation reactions with liver $\$ 9$ fraction}

Reactions to test and optimise steroid sulfation were prepared with final concentrations of: $50 \mathrm{mM}$ Tris. $\mathrm{HCl}$ for $\mathrm{pH} 7.4,0.55 \mathrm{mg} \mathrm{mL}^{-1}$ total protein from equine liver $\mathrm{S} 9$ fraction, $30 \mu \mathrm{M}$ steroid, $5 \mathrm{mM} \mathrm{MgCl}_{2}$, and various sulfation reagents. Nandrolone sulfate (NS) was used as an internal standard with a final concentration of $4 \mu \mathrm{M}$. 
Sulfation reagents consisted of: 80 or $200 \mu \mathrm{M}$ PAPS, or ATP and $\mathrm{Na}_{2} \mathrm{SO}_{4}$ at a range of final concentrations. The ratio of ATP to sulfate was kept at 2:1, in keeping with the stoichiometry for PAPS synthesis (Figure 1). The final ATP concentration was tested from 2 to $32 \mathrm{mM}$ with EA as a substrate to demonstrate 16 $\mathrm{mM}$ as optimal (Supporting Information Figure S1). The optimal $\mathrm{MgCl}_{2}$ concentration was then determined to be $20 \mathrm{mM}$ with $16 \mathrm{mM}$ ATP and $8 \mathrm{mM}$ sulfate (Supporting Information Figure S2).

Reactions were started by adding PAPS or sulfation reagent, with a final volume of $400 \mu \mathrm{L}$, and incubated at $37^{\circ} \mathrm{C}$. Aliquots of $50 \mu \mathrm{L}$ were taken from reactions or standards at defined time points and quenched by mixing with $150 \mu \mathrm{L}$ of $66 \%(\mathrm{v} / \mathrm{v})$ methanol in water. The quenched sample was centrifuged at 21000 $g$ for $10 \mathrm{~min}$, the supernatant transferred to a new vial and $20 \mu \mathrm{L}$ injected for LC-MS analysis (section 2.2.1). The EAS peaks were integrated with ChemStation software (Agilent), and the response ratio of EAS to NS (internal standard) was determined and compared to the response ratio for a $30 \mu \mathrm{M}$ EAS external standard.

\subsubsection{Comparison among species and steroids}

To test the general utility of ATP and $\mathrm{Na}_{2} \mathrm{SO}_{4}$ as a source of PAPS the amount of sulfation for six steroids at $30 \mu \mathrm{M}$ concentration: A, EA, EC, E, T and ET was tested. These were tested with three different liver S9 fractions: human, canine and equine. Reactions and analysis were performed as described above (section 2.3.1), except that the optimised concentrations were used for ATP (16 mM), sulfate (8 mM) and $\mathrm{MgCl}_{2}$ (20 mM). Reactions were performed in triplicate and were incubated at $37^{\circ} \mathrm{C}$ for 24 hours. Steroid sulfate peaks were integrated with ChemStation software, and the response ratio of steroid sulfate to NS (internal standard) was determined and compared to the response ratio for $30 \mu \mathrm{M}$ steroid sulfate external standard.

\subsubsection{In vitro phase II metabolism of furazadrol using PAPS}

In vitro phase II metabolism was performed by modification of a reported method.25 A solution containing steroid $(120 \mu \mathrm{M}, 250 \mu \mathrm{L})$ in sodium phosphate buffer (100 mM, pH 7.4) and methanol (0.4\%) was treated in order with the following solutions: aqueous magnesium chloride (1.0 M, $2.3 \mu \mathrm{L})$, aqueous G6P (100 mM, $37.5 \mu \mathrm{L})$, aqueous NAD (50 mM, $15 \mu \mathrm{L}$ ), aqueous NADP (50 mM, $15 \mu \mathrm{L})$, aqueous NADdependant G6PDH (40 units $\mathrm{mL}^{-1}, 12.5 \mu \mathrm{L}$ ), aqueous NADP-dependant G6PDH (40 units $\mathrm{mL}^{-1}, 12.5 \mu \mathrm{L}$ ), aqueous PAPS (1.6 mM, $22.5 \mu \mathrm{L})$, additional aqueous magnesium chloride in association with PAPS (1.0 M, $2.5 \mu \mathrm{L})$, aqueous UDPGA $(610 \mu \mathrm{M}, 31 \mu \mathrm{L})$, water $(74 \mu \mathrm{L})$, and equine liver S9 fraction (20 mg mL-1, 25 $\mu \mathrm{L})$. The final solution $(500 \mu \mathrm{L})$ was then incubated in an open tube with agitation for $16 \mathrm{~h}$ at $37^{\circ} \mathrm{C}$. The reaction was quenched with acetonitrile $(1 \mathrm{~mL})$, centrifuged (2000 rpm, $5 \mathrm{~min}$ ) to pellet solids, and the supernatant was decanted. Concentration of the supernatant under a stream of nitrogen at $60{ }^{\circ} \mathrm{C}$ afforded a residue which was reconstituted in methanol-water (5:95 v/v, $200 \mu \mathrm{L})$ and transferred to a sealed vial for subsequent LC-MS analysis as per section 2.2. Control experiments excluding PAPS, UDPGA, both UDPGA and PAPS, all phase I co-factors, equine liver S9 fraction, and steroid respectively 
were performed alongside the above reaction, with addition of water or buffer as required to maintain a constant final reaction volume and buffer concentration.

\subsubsection{In vitro phase II metabolism of furazadrol and superdrol using ATP and $\mathrm{Na}_{2} \mathrm{SO}_{4}$}

In vitro metabolism with ATP and $\mathrm{Na}_{2} \mathrm{SO}_{4}$ was performed as per section 2.3.3 with the following substitutions: the PAPS solution was replaced by addition of aqueous ATP (250 mM, $32 \mu \mathrm{L}$ ), aqueous $\mathrm{Na}_{2} \mathrm{SO}_{4}(100 \mathrm{mM}, 40 \mu \mathrm{L})$, and additional aqueous magnesium chloride $(1.0 \mathrm{M}, 5.7 \mu \mathrm{L})$. The volume of water added was reduced to maintain a constant final reaction volume (500 $\mu \mathrm{L})$. Control experiments analogous to those reported in section 2.3.3 were also performed alongside this reaction.

\section{Results and Discussion}

\subsection{Steroid sulfation with liver S9 fraction, ATP and sodium sulfate}

Sulfation of EA was examined to test the hypothesis that stable and readily available reagents, ATP and $\mathrm{Na}_{2} \mathrm{SO}_{4}$, could be added to liver S9 fraction leading to PAPS synthesis and SULT-catalysed sulfation. Sulfation was detected as EAS production and the concentrations of $\mathrm{ATP}, \mathrm{Na}_{2} \mathrm{SO}_{4}$ and $\mathrm{MgCl}$ were subsequently optimised (with ATP: $\mathrm{Na}_{2} \mathrm{SO}_{4}$ fixed at 2:1) as 16, 8, and $20 \mathrm{mM}$ respectively (section 2.3.1). This optimised reaction was then compared with reactions using PAPS at 80 or $200 \mu \mathrm{M}$ with $5 \mathrm{mM} \mathrm{MgCl} 2$ (Figure 3). Equine liver $\mathrm{S} 9$ fraction, ATP and $\mathrm{Na}_{2} \mathrm{SO}_{4}$ displayed steady EAS production over the first six hours, with continued EAS production but declining rates up to 24 hours. Meanwhile, $80 \mu \mathrm{M}$ PAPS afforded a similar initial rate over the first six hours but EAS production ceased thereafter. Productivity with PAPS was not greatly enhanced by increased concentration; $200 \mu \mathrm{M}$ PAPS gave the lowest initial rate and only resulted in a slight increase in EAS production relative to $80 \mu \mathrm{M}$ PAPS after 24 hours.

The three conditions of ATP and $\mathrm{Na}_{2} \mathrm{SO}_{4}, 80 \mu \mathrm{M}$ and $200 \mu \mathrm{M}$ PAPS produced approximately 15, 6 and 7.5 $\mu \mathrm{M}$ EAS respectively after $24 \mathrm{~h}$, with ATP and $\mathrm{Na}_{2} \mathrm{SO}_{4}$ displaying the greatest EAS production beyond six hours. However, even after 24 hours, this reaction only reached about 50\% completion. In all cases excess sulfation reagents were insufficient to generate $30 \mu \mathrm{M}$ EAS over $24 \mathrm{~h}$ and aside from limited time this could be due to competing substrates for SULTs present in the liver S9 fraction, PAPS instability or inactivation for SULTs over the course of the incubations. Alternatively, the PAPS precursor ATP may be subject to other biochemical transformations, thus limiting the PAPS synthesis and sulfation. Substrate and product inhibition also play a role in the reaction profiles. Substrate inhibition of human SULT2A1 has been observed for PAPS at a saturating concentration of dehydroepiandrosterone (DHEA), an unsaturated congener of EA. ${ }^{44}$ Although this phenomenon is highly dependent on DHEA concentration, it could be the basis for the lower initial rates observed for $200 \mu \mathrm{M}$ PAPS compared with $80 \mu \mathrm{M}$ PAPS for EAS production. 


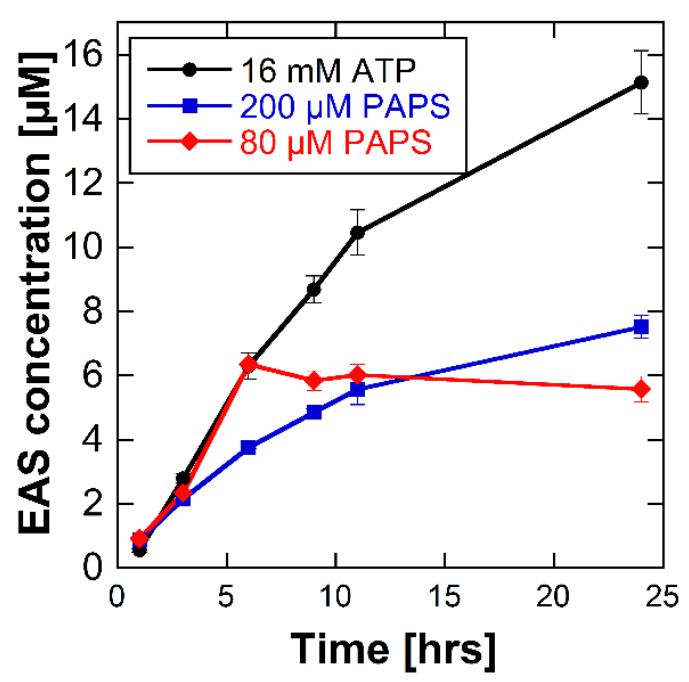

Figure 3: A temporal analysis of EAS production in vitro using equine S9 fraction and different phase II cofactors. Reactions were performed as described in section 2.3.1 with $30 \mu \mathrm{M}$ EA and either: $80 \mu \mathrm{M}$ PAPS (red points), $200 \mu \mathrm{M}$ PAPS (blue points), or $16 \mathrm{mM} \mathrm{ATP,} 8 \mathrm{mM} \mathrm{Na}_{2} \mathrm{SO}_{4}$ and $20 \mathrm{mM} \mathrm{MgCl}_{2}$ (black points) as PAPS precursor. The error bars represent the standard deviation from three independent reactions.

The general applicability of ATP and $\mathrm{Na}_{2} \mathrm{SO}_{4}$ for in vitro PAPS synthesis and steroid sulfation was demonstrated for the six steroids and equine, human and canine S9 fractions (Figure 4). There were distinct differences among the three species for steroid sulfation. Both human and equine systems were capable of sulfating all six steroids with peak activity for EAS with equine and ETS with human S9 fractions. Production of ES was lowest for the S9 fractions from both these species. This result reflects the expression profile for human liver where the SULT responsible for androgen sulfation (SULT2A1) predominates and that for estradiol (SULT1E1) is only a minor component. ${ }^{36,37}$ In contrast, canine S9 fraction had greatest activity for ES and no detectable activity for ECS, TS or ETS production. This observation is in agreement with previous studies into canine metabolism where glucuronylation is the predominant form of phase II conjugation for steroids with sulfation making only a minor contribution. ${ }^{3}$ Furthermore, the major SULT expressed in canine liver, SULT1A1, has been expressed recombinantly and shown to sulfate estradiol, but not DHEA. In summary, ATP and $\mathrm{Na}_{2} \mathrm{SO}_{4}$ gave sulfate ester synthesis for a range of steroids using the liver $\mathrm{S} 9$ fractions derived from humans, horses and dogs and therefore serves as a suitable replacement for PAPS for in vitro metabolism studies. Attention then turned to the use of ATP and $\mathrm{Na}_{2} \mathrm{SO}_{4}$ as a replacement of PAPS for the in vitro metabolism of the synthetic steroids $\mathbf{F}$ and $\mathbf{S}$. 


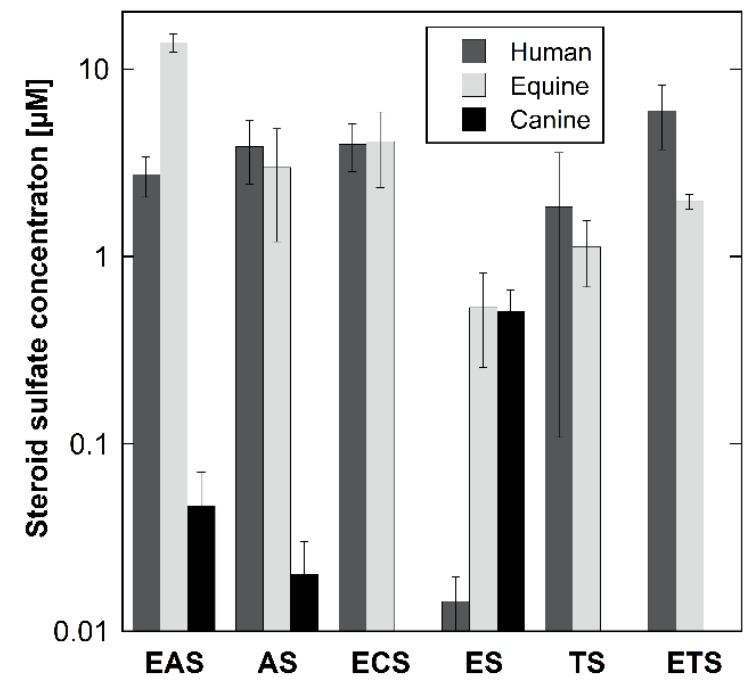

Figure 4 Comparing sulfation of six different steroids (30 $\mu \mathrm{M}$, indicated on the x-axis) with liver S9 fraction from three different species: human (dark grey), equine (light grey), and canine (black). Reactions were performed as described in section 2.3.2 with ATP and $\mathrm{Na}_{2} \mathrm{SO}_{4}$ as precursors for PAPS. The production of each steroid sulfate $(\mu \mathrm{M})$ is presented on a logarithmic scale with the error bars indicating the standard deviation from three independent reactions.

\subsection{Synthesis of steroid reference materials}

The synthesis of the furazadrol reference materials used in this study has been described in a previous publication. ${ }^{25}$ A number of phase I and II superdrol reference were prepared based on predicted patterns of equine metabolism ${ }^{2}$ or the phase I metabolites tentatively assigned in a previous equine in vitro metabolism study. ${ }^{5}$ The reduction of $\mathbf{S}$ with sodium borohydride gave rise to a mixture of $3 \alpha / \beta$ hydroxy isomers $\mathbf{3} \boldsymbol{\alpha}$-RS and $\mathbf{3} \boldsymbol{\beta}$-RS, favouring the $3 \boldsymbol{\beta}$-isomer $\mathbf{3} \boldsymbol{\beta}$-RS. ${ }^{45}$ On the other hand, reduction with L-Selectride $₫$, which is a bulky reducing agent, exclusively gave rise to the $3 \alpha$-alcohol isomer $\mathbf{3} \boldsymbol{\alpha}$-RS. Performing these reactions on the milligram-scale proceeded smoothly but did not allow these isomers to be readily separated. As a result, the $3 \beta$-isomer $\mathbf{3} \boldsymbol{\beta}$-RS was prepared with a minor $3 \alpha$-impurity $\mathbf{3} \boldsymbol{\alpha}$ $\mathbf{R S}$, which was carried through subsequent reaction steps. The pure $3 \alpha$-isomer $\mathbf{3} \boldsymbol{\alpha}$-RS was used to identify the presence of minor $3 \alpha$-isomer products generated during reactions of the $3 \beta$-isomer $\mathbf{3} \boldsymbol{\beta}$-RS. Mono-sulfation of the $3 \alpha / \beta, 17 \beta$-diols to give $\mathbf{3} \boldsymbol{\alpha}$-RSS, and mixed $\mathbf{3} \boldsymbol{\alpha}$-RSS/3 $\boldsymbol{\beta}$-RSS proceeded in high yield, using established methodology.40 0 the other hand, enzymatic glucuronylation afforded only the $3 \beta$-isomer $\mathbf{3} \boldsymbol{\beta}$-RSG. ${ }^{41}$ This was highlighted by the reaction of a mixture of $3 \alpha / \beta$-hydroxy isomers $\mathbf{3} \boldsymbol{\alpha}$ RS/3 $\beta$-RS, which afforded $2 \alpha, 17 \alpha$-dimethyl-5 $\alpha$-androstane-3 $\beta, 17 \beta$-diol 3 -glucuronide $\mathbf{3} \beta$-RSG as the sole product after purification by SPE. Unreacted $\mathbf{3} \boldsymbol{\alpha}-\mathbf{R S}$ was isolated from the reaction mixture. The selectivity for the glucuronylation of $3 \beta$-hydroxy steroids using this enzyme has been documented, and presumably reflects the substrate binding within the enzyme active site. ${ }^{41}$ 


\subsection{In vitro phase II metabolism of furazadrol}

In vitro studies typically involve only phase I metabolism, and phase II metabolism is not routinely explored, despite the fact that for in vivo studies, phase II conjugates are usually the major metabolites observed.1,2,3 The addition of phase II co-factors has the potential to modify phase I metabolism by selectively intercepting phase I intermediates, and as a result, the inclusion of in vitro phase II metabolism has the potential to generate a more detailed metabolic profile that can better match the in vivo profile.

To explore these issues, the phase I and phase II equine metabolism of the synthetic steroid $\mathbf{F}$ was investigated. This compound was chosen as it allowed comparison to a recently reported in vivo equine administration study, and a comparative phase I in vitro study (F:IF 9:1). ${ }^{25}$ In this work, the metabolism reactions were conducted as previously reported with minor variations. ${ }^{25}$ Together with the addition of phase II cofactors UDPGA and PAPS or ATP and sodium sulfate, the phase I cofactors NADH and NADPH were regenerated using G6P and appropriate G6PDH enzymes. Metabolism data were examined using mass filters for predicted metabolites formed from up to three metabolic transformations including oxidation, reduction and hydroxylation, with or without subsequent sulfation or glucuronylation. Metabolite peaks were identified where exact masses were observed within \pm 10 ppm of the predicted mass, and by comparison with control experiments. Metabolites were also matched against synthesised reference materials where available. A comparison of the previously reported study 25 with the present study is outlined below (Table 1). A table of the observed metabolites, retention times, precursor ions and MS/MS fragments are reported in the Supporting Information, together with copies of extracted ion chromatograms and MS/MS spectra.

\section{Table 1}

The in vitro metabolism of furazadrol (F:IF 9:1) was conducted using both UDPGA with PAPS, and UDPGA with ATP and $\mathrm{Na}_{2} \mathrm{SO}_{4}$ (Table 1). Employing UDPGA and PAPS, major phase II metabolites were observed including FS, IFS, FG, IFG, and EFG, which were matched to reference materials. Additionally, EFS was not observed by comparison to the reference material, identical to observations from the in vivo study. ${ }^{25}$ Minor unidentified phase II metabolites were observed including six hydroxylated furazadrol sulfate metabolites (S1-S6), one oxidised and hydroxylated furazadrol sulfate metabolite (S7), five hydroxylated furazadrol glucuronide metabolites (G1-G4, G6), and two oxidised and hydroxylated furazadrol glucuronide metabolites (G9-G10). Of these minor metabolites, S2 was identified as a match with the previously reported in vivo study. ${ }^{25}$ On the other hand, the in vitro phase II study employing UDPGA, ATP and $\mathrm{Na}_{2} \mathrm{SO}_{4}$, identified the same major phase II metabolites including FS, IFS, FG, IFG, and EFG. Again EFS was not observed. Minor unidentified phase II metabolites were observed including six hydroxylated furazadrol sulfate metabolites (S1-S6), one oxidised and hydroxylated furazadrol sulfate metabolite (S8), two hydroxylated furazadrol glucuronide metabolites (G3, G5), and three oxidised and hydroxylated furazadrol glucuronide metabolites (G7, G8, G10). Of 
these minor metabolites, $\mathbf{S 2}$, and $\mathbf{S 8}$ were identified as matches with the previously reported in vivo study. ${ }^{25}$

A comparison of the phase II metabolites observed using both PAPS or ATP and $\mathrm{Na}_{2} \mathrm{SO}_{4}$ in vitro systems (Table 1) indicated that a majority of the metabolites were common. The major phase II metabolites FS, IFS, FG, IFG, and EFG were common to both systems and in both instances EFS was not observed. Thus the in vitro studies clearly indicate that EF serves as substrate for UGT promoted glucuronylation but not SULT promoted sulfation. This could arise due to different enzyme-substrate affinities or reaction rates for the competing conjugation processes. It is consistent with observed patterns of equine in vivo metabolism where $17 \alpha$-hydroxy metabolites are commonly found in the glucuronide, but not the sulfate, fraction. ${ }^{2}$

Although the major phase II metabolites were common to both in vitro systems, the minor phase II metabolites appeared to vary between systems, particularly for the glucuronides (S1-S6, G3, G10 were common, S7, G1, G2, G4, G6, G9 PAPS only, S8, G5, G7, G8 ATP and $\mathrm{Na}_{2} \mathrm{SO}_{4}$ only). The differences in the glucuronide profile may be attributed to the different sulfation reagents used in these systems. In the experiment utilising $80 \mu \mathrm{M}$ PAPS, the co-factors are added at the start of the metabolism reaction, and PAPS is then consumed or decays in solution over time (Figure 3). This results in a burst of phase II sulfation towards the beginning of the reaction, which ceases relatively quickly. This is in contrast to the experiments utilising ATP and $\mathrm{Na}_{2} \mathrm{SO}_{4}$ in which a relatively steady rate of sulfation was observed. Of particular interest were the minor metabolites $\mathbf{S 2}$ and $\mathbf{S 8}$, which were both observed in the previous in vivo study. ${ }^{25}$ The in vitro phase II study employing PAPS identified $\mathbf{S} 2$ only, while the study employing ATP and $\mathrm{Na}_{2} \mathrm{SO}_{4}$ identified both $\mathbf{S} 2$ and $\mathbf{S 8}$ as minor metabolites, suggesting that in vitro platforms employing ATP and $\mathrm{Na}_{2} \mathrm{SO}_{4}$ could offer some advantages for generating some phase II metabolites.

A comparison of the phase I metabolites observed using both PAPS, and ATP/ $\mathrm{Na}_{2} \mathrm{SO}_{4}$ in vitro systems indicated that a majority of the metabolites were common (EF, OF, OIF, M2-M5, M8, M9, M12, M13, M15, M16), with a few exceptions (M1, M10, M14 PAPS only; M6, M7, M11 ATP and $\mathrm{Na}_{2} \mathrm{SO}_{4}$ only). The number of phase I metabolites observed did not appear to change significantly in control experiments where phase II co-factors were excluded, suggesting that phase II metabolism was not fully intercepting the phase I metabolites. The extent of phase I metabolism in the present study appeared to be somewhat greater than reported previously. ${ }^{25}$ The present study used a combination of NADH and NADPH cofactors with G6P and both E. coli and L. mesenteroides G6PDH enzymes for regeneration of both. In contrast, the previous study used NADH G6P and L. mesenteroides G6PDH. Although liver extracts enable $\mathrm{NADH}$ to reduce $\mathrm{NADP}^{+}$and vice versa, additional NADPH for cytochrome P450 oxidation could explain the modest increase in the number of hydroxylated metabolites identified in the present study. For both systems, metabolites, EF, OF, OIF, M2-M4, and M8 were identified as matches with the phase I study reported previously. ${ }^{25}$ Although no unconjugated metabolites were identified in the previous in vivo 
study,25 the hydroxylated furazadrol M4 was observed to match a phase I metabolite generated after Pseudomonas aeruginosa arylsulfatase hydrolysis of the in vivo samples. ${ }^{25} 43$

It appears that the substitution of PAPS with ATP and $\mathrm{Na}_{2} \mathrm{SO}_{4}$ is capable of generating comparable in vitro metabolic profiles, and can serve as an economical alternative for the generation of in vitro phase II sulfate conjugates. The major metabolites FS, IFS, FG, IFG, and EFG, which include the most useful markers for anti-doping screening 25 were all detected at higher levels (response ratios 10-103 times) than the minor phase II metabolites in these studies. On the other hand, the minor in vivo metabolites S2, and $\mathbf{S 8}$ identified in the in vitro study would likely be difficult to distinguish from the other minor phase II metabolites observed. The long-term metabolites most suitable for use as screening markers are not always the most abundant, ${ }^{2}$ and so may be difficult to identify solely from in vitro data. Although it could be argued from these results that the reported conditions are capable of generating an adequate representation of the major in vivo metabolites, further work refining these conditions to better match the complete in vivo profile solely from in vitro results is likely to be beneficial. For equine and canine sports, AORC criteria allow for the use of in vitro-derived materials as reference materials in confirmatory analysis. ${ }^{21}$ Improved methods to mimic the in vivo profile using in vitro techniques will generate new reference materials and allow additional instances of AAS misuse to be detected and confirmed.

\subsection{In vitro phase II metabolism of superdrol}

Given the success of investigations into the metabolism of $\mathbf{F}$, attention turned to the equine in vitro phase II metabolism of $\mathbf{S}$. The equine in vivo metabolism of $\mathbf{S}$ has not been previously reported and in the absence of this information this study serves as a prediction of the likely metabolic profile of this steroidal agent. Previous reports of the equine in vitro phase I metabolism of $\mathbf{S}$ provided some comparison with the present study. ${ }^{5}$ Several human in vivo and in vitro studies of $\mathbf{S}$ metabolism have also been reported. ${ }^{46,47,48,49}$ Metabolism of $\mathbf{S}$ with UDPGA, ATP, $\mathrm{Na}_{2} \mathrm{SO}_{4}$, and equine liver S9 fraction afforded a range of phase II sulfate and glucuronide metabolites which are summarised in Figure 5. A table of the observed metabolites, retention times, precursor ions and MS/MS fragments are reported in the Supporting Information, together with copies of extracted ion chromatograms and MS/MS spectra. 


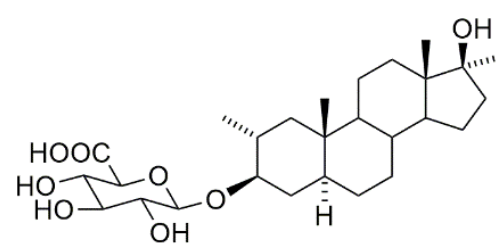

3 $\beta$-RSG ${ }^{A}$
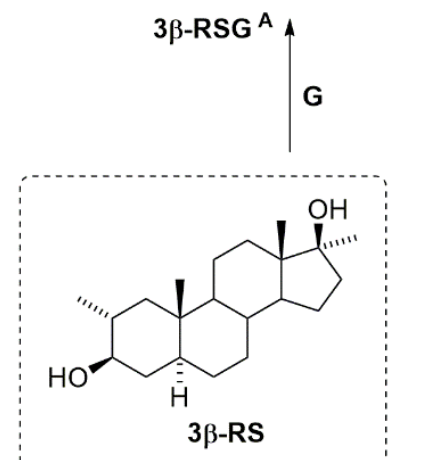

S

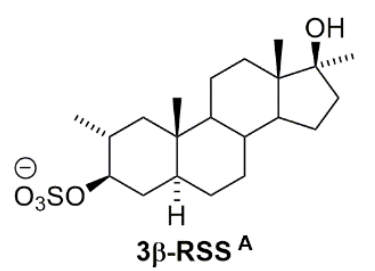

$3 \beta-$ RSS $^{\mathrm{A}}$
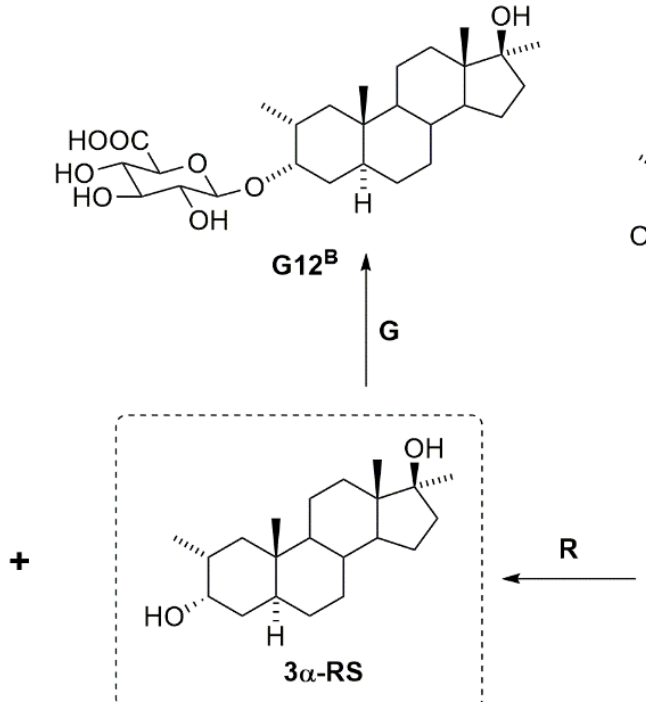

H

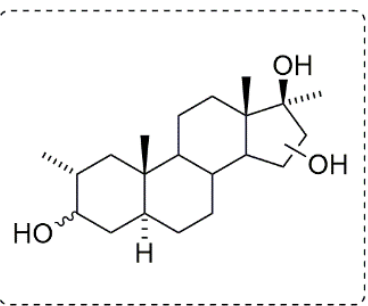

R $\mathbf{S}$

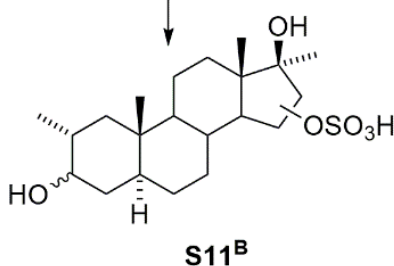

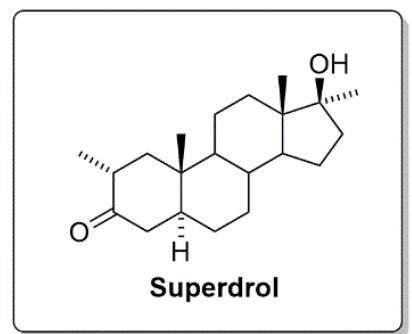

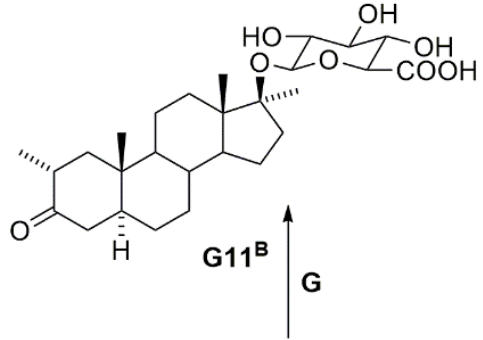

H

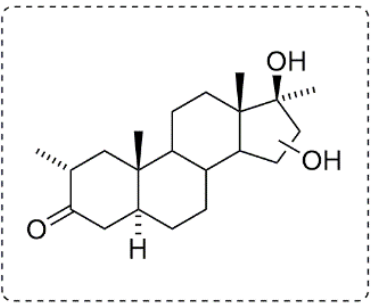

S

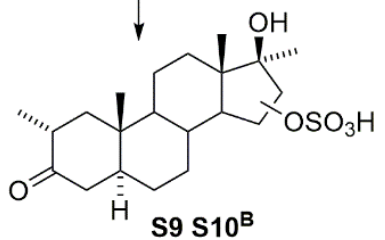

Figure 5: Proposed in vitro equine metabolism of superdrol (S); ${ }^{A}$ Matched to reference material; BUnconfirmed regio- and stereo-chemistry. $\mathrm{H}$, hydroxylation; $\mathrm{R}$, reduction; G, glucuronylation; $\mathrm{S}$, sulfation. Dotted boxes indicate presumed intermediates that were not observed in this LC-MS study.

Major phase II metabolites were observed including 3及-RSS which was matched to the available reference material, and a reduced superdrol glucuronide metabolite G12. ${ }^{48,49}$ Although no reference material was available, G12 was tentatively assigned as $2 \alpha, 17 \alpha$-dimethyl-5 $\alpha$-androstane-3 $\alpha, 17 \beta$-diol 3glucuronide (or its 17-glucuronide regioisomer), as this metabolite did not match the reference material for 3 $\beta$-RSG but exhibited similar mass spectrometry behaviour including the proton loss species $\mathrm{m} / \mathrm{z}$ $495\left([\mathrm{M}-\mathrm{H}]^{-}\right)$and the glucuronide derived fragments $m / z 113\left(\mathrm{C}_{5} \mathrm{H}_{5} \mathrm{O}_{3}^{-}\right), \mathrm{m} / z 85\left(\mathrm{C}_{4} \mathrm{H}_{5} \mathrm{O}_{2^{-}}\right)$and $\mathrm{m} / z 75$ $\left(\mathrm{C}_{2} \mathrm{H}_{3} \mathrm{O}_{3}-{ }^{-}{ }^{50} \mathrm{~A}\right.$ metabolite corresponding to $2 \alpha, 17 \alpha$-dimethyl-5 $\alpha$-androstane-3 $\alpha, 17 \beta$-diol 3-glucuronide (or its 17-glucuronide regioisomer) has also been indirectly observed in human in vivo and in vitro studies of $\mathbf{S}$ metabolism. ${ }^{48,49}$ Despite this, some caution is required as the formation of stereoisomeric 
$17 \beta$-methyl-17 $\alpha$-hydroxy metabolites through formation and hydrolysis of the tertiary sulfate conjugate provides alternative metabolic pathways. ${ }^{18,19,20}$ Minor phase II metabolites were observed including two hydroxylated superdrol sulfate metabolites $(\mathbf{S 9}, \mathbf{S 1 0})$, a reduced and hydroxylated superdrol sulfate metabolite (S11), superdrol glucuronide (G11), 3及-RSG, and two reduced and hydroxylated superdrol glucuronide metabolites $(\mathbf{G 1 3}, \mathbf{G 1 4})$. Although the majority of minor phase II metabolites remain unidentified in this study, the minor metabolite 3 $\beta$-RSG was confirmed, and $\mathbf{3} \boldsymbol{\alpha}$-RSS was not observed, by comparison to the corresponding reference materials.

The formation of the $3 \beta$-hydroxy metabolites is known to be favoured in horses, ${ }^{2}$ and this is followed by sulfation to give major $\mathbf{3} \boldsymbol{\beta}$-RSS and glucuronylation to give minor $\mathbf{3} \boldsymbol{\beta}$-RSG. Alternatively, the tentative assignment above suggests that formation of the $3 \alpha$-hydroxy metabolite $\mathbf{3} \boldsymbol{\alpha}$-RS is followed by glucuronylation to give major $\mathbf{G 1 2}{ }^{48,49}$ but not sulfation to $3 \boldsymbol{\alpha}$-RSS, in a pattern similar to that observed in humans. ${ }^{1}$ Although no unconjugated phase I metabolites were identified directly in this LC-MS study, the phase I metabolism of $\mathbf{S}$ is known to afford a number of steroid diol, and triol metabolites which ionise poorly under +ESI conditions. ${ }^{5}$ Instead, these metabolites were identified indirectly through analysis of their intact phase II conjugates. Future studies of the in vivo metabolism of $\mathbf{S}$ are likely to benefit from access to a wider variety of reference materials and the application of both GC-MS and LCMS for sample analysis. Overall, the phase I metabolism of $\mathbf{S}$ appears to be quite simple, and the major phase II conjugates observed correlate well with the major phase I metabolites previously reported for the equine in vitro studies. ${ }^{5}$ The reduced superdrol isomers $\mathbf{3} \boldsymbol{\beta}-\mathbf{R S}$, and $\mathbf{3} \boldsymbol{\alpha}-\mathbf{R S}$ appear to be the most abundant in vitro metabolites observed, and would likely be among the major metabolites observed in vivo. Additionally, these compounds are also easily prepared from the parent compound, and so should be available as reference materials to assist in confirmation. As such, it is recommended that anti-doping

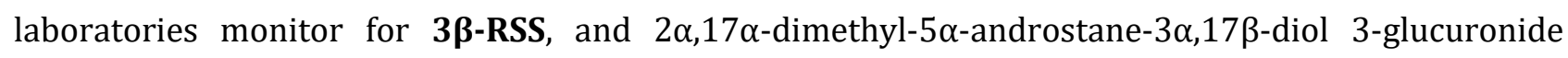
(tentatively identified as $\mathbf{G 1 2}$ ), or their phase I counterparts $\mathbf{3} \boldsymbol{\beta}-\mathbf{R S}$, and $\mathbf{3} \boldsymbol{\alpha}-\mathbf{R S}$, until such time as a comparative in vivo study can be undertaken. 


\section{Conclusion}

In this work alternative conditions for in vitro phase II sulfation using human, equine or canine liver S9 fraction were developed and employed for the generation of six representative steroidal sulfates, with ATP and $\mathrm{Na}_{2} \mathrm{SO}_{4}$ in place of the expensive and unstable co-factor PAPS. Using these conditions, the equine in vitro phase II metabolism of the synthetic steroid $\mathbf{F}$ was investigated, with ATP and $\mathrm{Na}_{2} \mathrm{SO}_{4}$ found to offer comparable metabolism to reactions using PAPS. The major in vitro metabolites of $\mathbf{F}$ also correlated closely with the metabolites observed in a previously reported equine in vivo study. ${ }^{25}$ Although these in vitro methods do not fully replicate the in vivo metabolic profile, it is expected that future work will refine these methods to more accurately predict the metabolism of new compounds from in vitro studies. Finally, the equine in vitro phase II metabolism of the synthetic steroid $\mathbf{S}$ was also performed that serves as a prediction of the in vivo metabolic profile. Notably the equine in vitro phase II metabolites observed in this study correspond well with previously reported equine in vitro phase I studies and so are recommended as suitable screening markers until in vivo studies can be undertaken.

\section{Acknowledgements}

The authors would like to thank the Australian Research Council's Linkage Projects funding scheme (LP120200444 - Strategies for the detection of designer steroids in racehorses) for financial support, and Ms Candace Greer, Ms Lauren McClure and Ms Corrine Smart at the Australian Racing Forensic Laboratory (Sydney, Australia) for technical assistance with LC-MS analysis.

\section{References}

(1) Schänzer, W. Metabolism of anabolic androgenic steroids. Clin. Chem. 1996, 42 (7), 1001-1020.

(2) Scarth, J. P.; Teale, P.; Kuuranne, T. Drug metabolism in the horse: a review. Drug Test. Anal. 2011, 3 (1), 19-53.

(3) McKinney, A. R.; Cawley, A. T.; Young, E. B.; Kerwick, C. M.; Cunnington, K.; Stewart, R. T.; Ambrus, J. I.; Willis, A. C.; McLeod, M. D. The metabolism of anabolic-androgenic steroids in the greyhound. Bioanalysis 2013, 5 (7), 769-781.

(4) Brandon, E. F. A.; Raap, C. D.; Meijerman, I.; Beijnen, J. H.; Schellens, J. H. M. An update on in vitro test methods in human hepatic drug biotransformation research: pros and cons. Toxicol. Appl. Pharmacol. 2003, 189 (3), 233-246.

(5) Clarke, A.; Scarth, J.; Teale, P.; Pearce, C.; Hillyer, L. The use of in vitro technologies and highresolution/accurate-mass LC-MS to screen for metabolites of 'designer' steroids in the equine. Drug Test. Anal. 2011, 3 (1), 74-87.

(6) Joseph, J. F.; Parr, M. K. Synthetic androgens as designer supplements. Curr. Neuropharmacol. 2015, 13 (1), 89-100.

(7) Waller, C. C.; McLeod, M. D. A review of designer anabolic steroids in equine sports. Drug Test. Anal. 2016, in press, DOI: $10.1002 /$ dta.2112.

(8) Deventer, K.; Pozo, O. J.; Verstraete, A. G.; Van Eenoo, P. Dilute-and-shoot-liquid chromatographymass spectrometry for urine analysis in doping control and analytical toxicology. TrAC Trends Anal. Chem. 2014, 55, 1-13.

(9) Gómez, C.; Pozo, O. J.; Marcos, J.; Segura, J.; Ventura, R. Alternative long-term markers for the detection of methyltestosterone misuse. Steroids 2013, 78 (1), 44-52. 
(10) Strahm, E.; Baume, N.; Mangin, P.; Saugy, M.; Ayotte, C.; Saudan, C. Profiling of 19-norandrosterone sulfate and glucuronide in human urine: Implications in athlete's drug testing. Steroids 2009, 74 (3), 359-364.

(11) Torrado, S.; Roig, M.; Farré, M.; Segura, J.; Ventura, R. Urinary metabolic profile of 19-norsteroids in humans: glucuronide and sulphate conjugates after oral administration of 19-nor-4androstenediol. Rapid Commun. Mass Spectrom. 2008, 22 (19), 3035-3042.

(12) Gómez, C.; Pozo, O. J.; Garrostas, L.; Segura, J.; Ventura, R. A new sulphate metabolite as a longterm marker of metandienone misuse. Steroids 2013, 78 (12-13), 1245-1253.

(13) Thevis, M.; Piper, T.; Horning, S.; Juchelka, D.; Schänzer, W. Hydrogen isotope ratio mass spectrometry and high-resolution/high-accuracy mass spectrometry in metabolite identification studies: Detecting target compounds for sports drug testing. Rapid Commun. Mass Spectrom. 2013, 27 (17), 1904-1912.

(14) Liu, Y.; Lu, J.; Yang, S.; Xu, Y.; Wang, X. A new potential biomarker for 1-testosterone misuse in human urine by liquid chromatography quadruple time-of-flight mass spectrometry. Anal. Methods 2015, 7 (11), 4486-4492.

(15) Gómez, C.; Pozo, O. J.; Geyer, H.; Marcos, J.; Thevis, M.; Schänzer, W.; Segura, J.; Ventura, R. New potential markers for the detection of boldenone misuse. J. Steroid Biochem. Mol. Biol. 2012, 132 (3-5), 239-246.

(16) Boccard, J.; Badoud, F.; Grata, E.; Ouertani, S.; Hanafi, M.; Mazerolles, G.; Lantéri, P.; Veuthey, J.-L.; Saugy, M.; Rudaz, S. A steroidomic approach for biomarkers discovery in doping control. Forensic Sci. Int. 2011, 213 (1-3), 85-94.

(17) Piper, T.; Schänzer, W.; Thevis, M. Genotype-dependent metabolism of exogenous testosterone new biomarkers result in prolonged detectability. Drug Test. Anal. 2016, 8 (11-12), 1163-1173.

(18) Schänzer, W.; Opfermann, G.; Donike, M. 17-Epimerization of $17 \alpha$-methyl anabolic steroids in humans: metabolism and synthesis of $17 \alpha$-hydroxy-17 $\beta$-methyl steroids. Steroids 1992, 57 (11), 537-550.

(19) Bi, H.; Massé, R. Studies on anabolic steroids-12. Epimerization and degradation of anabolic $17 \beta-$ sulfate-17 $\alpha$-methyl steroids in human: Qualitative and quantitative GC/MS analysis. J. Steroid Biochem. Mol. Biol. 1992, 42 (5), 533-546.

(20) Bi, H.; Massé, R.; Just, G. Studies on anabolic steroids. 9. Tertiary sulfates of anabolic $17 \alpha$-methyl steroids: synthesis and rearrangement. Steroids 1992, 57 (7), 306-312.

(21) Association of Official Racing Chemists. AORC MS Criteria (modified 23 Aug 16) http://www.aorc-online.org/documents/aorc-ms-criteria-modified-23-aug-16/ (accessed Sep $8,2016)$.

(22) Duffus, J. H.; Nordberg, M.; Templeton, D. M. Glossary of terms used in toxicology, 2nd edition (IUPAC Recommendations 2007). Pure Appl. Chem. 2007, 79 (7), 1153-1344.

(23) Adenosine 3 '-phosphate 5'-phosphosulfate lithium salt hydrate A1651 http://www.sigmaaldrich.com/catalog/product/sigma/a1651 (accessed Mar 23, 2017).

(24) Burkart, M. D.; Izumi, M.; Chapman, E.; Lin, C.-H.; Wong, C.-H. Regeneration of PAPS for the Enzymatic Synthesis of Sulfated Oligosaccharides. J. Org. Chem. 2000, 65 (18), 5565-5574.

(25) Waller, C. C.; Cawley, A. T.; Suann, C. J.; Ma, P.; McLeod, M. D. In vivo and in vitro metabolism of the designer anabolic steroid furazadrol in thoroughbred racehorses. J. Pharm. Biomed. Anal. 2016, 124, 198-206.

(26) Kuuranne, T.; Pystynen, K.-H.; Thevis, M.; Leinonen, A.; Schänzer, W.; Kostiainen, R. Screening of in vitro synthesised metabolites of 4,9,11- trien-3-one steroids by liquid chromatography mass spectrometry. Eur. J. Mass Spectrom. 2008, 14 (3), 181.

(27) Wong, J. K. Y.; Chan, G. H. M.; Leung, D. K. K.; Tang, F. P. W.; Wan, T. S. M. Generation of phase II in vitro metabolites using homogenized horse liver. Drug Test. Anal. 2016, 8 (2), 241-247.

(28) Taylor, P.; Scarth, J. P.; Hillyer, L. L. Use of in vitro technologies to study phase II conjugation in equine sports drug surveillance. Bioanalysis 2010, 2, 1971-1988.

(29) Ping Wong, K.; Khoo, B. Y.; Sit, K. H. Biosynthesis of PAPS in vitro by human liver: Measurement by two independent assay procedures. Biochem. Pharmacol. 1991, 41 (1), 63-69.

(30) Lin, C.-H.; Shen, G.-J.; Garcia-Junceda, E.; Wong, C.-H. Enzymic Synthesis and Regeneration of 3'Phosphoadenosine 5'-Phosphosulfate (PAPS) for Regioselective Sulfation of Oligosaccharides. J. Am. Chem. Soc. 1995, 117 (30), 8031-8032. 
(31) Zhao, H.; van der Donk, W. A. Regeneration of cofactors for use in biocatalysis. Curr. Opin. Biotechnol. 2003, 14 (6), 583-589.

(32) Venkatachalam, K. Human 3'-phosphoadenosine 5'-phosphosulfate (PAPS) Synthase: Biochemistry, Molecular Biology and Genetic Deficiency. IUBMB Life 2003, 55 (1), 1-11.

(33) Burkart, M. D.; Izumi, M.; Chapman, E.; Lin, C.-H.; Wong, C.-H. Regeneration of PAPS for the enzymatic synthesis of sulfated oligosaccharides. J. Org. Chem. 2000, 65 (18), 5565-5574.

(34) Fuda, H.; Shimizu, C.; Lee, Y. C.; Akita, H.; Strott, C. A. Characterization and expression of human bifunctional 3'-phosphoadenosine 5'-phosphosulphate synthase isoforms. Biochem. J. 2002, 365 (2), 497-504.

(35) Schröder, E.; Gebel, L.; Eremeev, A. A.; Morgner, J.; Grum, D.; Knauer, S. K.; Bayer, P.; Mueller, J. W. Human PAPS Synthase Isoforms Are Dynamically Regulated Enzymes with Access to Nucleus and Cytoplasm. PLOS ONE 2012, 7 (1), e29559.

(36) Coughtrie, M. W. H. Function and organization of the human cytosolic sulfotransferase (SULT) family. Chem. Biol. Interact. 2016, 259, Part A, 2-7.

(37) Strott, C. A. Steroid Sulfotransferases. Endocr. Rev. 1996, 17 (6), 670-697.

(38) Sigma-Aldrich. Adenosine 5 -triphosphate disodium salt hydrate Grade I, $\geq 99 \%$, from microbial http://www.sigmaaldrich.com/catalog/product/sigma/a2383?lang=en\&region=US (accessed Feb 2, 2017).

(39) Martin, S. F.; Dodge, J. A. Efficacious modification of the Mitsunobu reaction for inversions of sterically hindered secondary alcohols. Tetrahedron Lett. 1991, 32 (26), 3017-3020.

(40) Waller, C. C.; McLeod, M. D. A simple method for the small scale synthesis and solid-phase extraction purification of steroid sulfates. Steroids 2014, 92, 74-80.

(41) Ma, P.; Kanizaj, N.; Chan, S.-A.; Ollis, D. L.; McLeod, M. D. The Escherichia coli glucuronylsynthase promoted synthesis of steroid glucuronides: improved practicality and broader scope. Org. Biomol. Chem. 2014, 12 (32), 6208-6214.

(42) Zakaria, N. A. Studies of glucose-6-phosphate dehydrogenase. PhD Thesis, Australian National University, 2016.

(43) Stevenson, B. J.; Waller, C. C.; Ma, P.; Li, K.; Cawley, A. T.; Ollis, D. L.; McLeod, M. D. Pseudomonas aeruginosa arylsulfatase: a purified enzyme for the mild hydrolysis of steroid sulfates. Drug Test. Anal. 2015, 7 (10), 903-911.

(44) Gulcan, H. O.; Duffel, M. W. Substrate inhibition in human hydroxysteroid sulfotransferase SULT2A1: Studies on the formation of catalytically non-productive enzyme complexes. Arch. Biochem. Biophys. 2011, 507 (2), 232-240.

(45) Mauli, R.; Ringold, H. J.; Djerassi, C. Steroids. CXLV. 2-Methylandrostane derivatives. Demonstration of the boat form in the bromination of $2 \alpha$-methylandrostan-17 $\beta$-ol-3-one. J. Am. Chem. Soc. 1960, 82, 5494-5500.

(46) Gauthier, J.; Goudreault, D.; Poirier, D.; Ayotte, C. Identification of drostanolone and 17methyldrostanolone metabolites produced by cryopreserved human hepatocytes. Steroids 2009, 74 (3), 306-314.

(47) Lootens, L.; Meuleman, P.; Leroux-Roels, G.; Van Eenoo, P. Metabolic studies with promagnon, methylclostebol and methasterone in the uPA+/+-SCID chimeric mice. J. Steroid Biochem. Mol. Biol. 2011, 127 (3-5), 374-381.

(48) Geldof, L.; Tudela, E.; Lootens, L.; van Lysebeth, J.; Meuleman, P.; Leroux-Roels, G.; van Eenoo, P.; Deventer, K. In vitro and in vivo metabolism studies of dimethazine. Biomed. Chromatogr. 2016, $30(8), 1202-1209$.

(49) Zhang, J.; Lu, J.; Wu, Y.; Wang, X.; Xu, Y.; Zhang, Y.; Wang, Y. New Potential Biomarker for Methasterone Misuse in Human Urine by Liquid Chromatography Quadrupole Time of Flight Mass Spectrometry. Int. J. Mol. Sci. 2016, 17 (10), 1628.

(50) Fabregat, A.; Pozo, O. J.; Marcos, J.; Segura, J.; Ventura, R. Use of LC-MS/MS for the open detection of steroid metabolites conjugated with glucuronic acid. Anal. Chem. 2013, 85 (10), 5005-5014. 
Table 1: Comparison of in vivo and in vitro (UDPGA and PAPS, or UDPGA, ATP and $\mathrm{Na}_{2} \mathrm{SO}_{4}$ ) metabolism of furazadrol (F:IF 9:1); ${ }^{\mathrm{A} S e e}$ ref 25; ${ }^{\mathrm{B}}$ /D not detected.

\begin{tabular}{|c|c|c|c|c|c|c|}
\hline Metabolic transformation & $\begin{array}{l}\text { In vivo } \\
\text { (previous } \\
\text { study) }\end{array}$ & $\begin{array}{l}\text { Phase II in vitro } \\
\text { (UDPGA and } \\
\text { PAPS) }\end{array}$ & $\begin{array}{l}\text { Phase II in vitro } \\
\text { (UDPGA, ATP, } \\
\text { and } \mathrm{Na}_{2} \mathrm{SO}_{4} \text { ) }\end{array}$ & $\begin{array}{l}\text { Phase I in vitro } \\
\text { (previous } \\
\text { study) }{ }^{\mathrm{A}}\end{array}$ & $\begin{array}{l}\text { Phase I in vitro } \\
\text { (UDPGA and } \\
\text { PAPS) }\end{array}$ & $\begin{array}{l}\text { Phase I in vitro } \\
\text { (UDPGA, ATP, } \\
\text { and } \mathrm{Na}_{2} \mathrm{SO}_{4} \text { ) }\end{array}$ \\
\hline $\begin{array}{l}\text { furazadrol } \\
\text { (major) }\end{array}$ & $\begin{array}{l}\text { FS, IFS, FG, IFG, } \\
\text { EFG }\end{array}$ & $\begin{array}{l}\text { FS, IFS, FG, IFG, } \\
\text { EFG }\end{array}$ & $\begin{array}{l}\text { FS, IFS, FG, IFG, } \\
\text { EFG }\end{array}$ & EF & $\mathbf{E F}$ & $\mathbf{E F}$ \\
\hline $\begin{array}{l}\text { oxidised furazadrol } \\
\text { (major) }\end{array}$ & $\mathrm{N} / \mathrm{D}^{\mathrm{B}}$ & $\mathrm{N} / \mathrm{D}^{\mathrm{B}}$ & $\mathrm{N} / \mathrm{D}^{\mathrm{B}}$ & OF, OIF & OF, OIF & OF, OIF \\
\hline $\begin{array}{l}\text { hydroxylated furazadrol } \\
\text { (minor) }\end{array}$ & sulfate (x 1) & $\begin{array}{l}\text { sulfate (x 6) } \\
\text { S1-S6 } \\
\text { glucuronide (x } \\
\text { 5) } \\
\text { G1-G4, G6 }\end{array}$ & $\begin{array}{l}\text { sulfate (x 6) } \\
\text { S1-S6 } \\
\text { glucuronide (x } \\
\text { 2) } \\
\text { G3, G5 }\end{array}$ & $(\times 8)$ & $\begin{array}{l}\text { (x 7) } \\
\text { M1-M5, M8, M9 }\end{array}$ & $\begin{array}{l}\text { (x 8) } \\
\text { M2-M9 }\end{array}$ \\
\hline $\begin{array}{l}\text { oxidised and hydroxylated furazadrol } \\
\text { (minor) }\end{array}$ & $\begin{array}{l}\text { sulfate (x 2) } \\
\text { glucuronide (x } \\
\text { 2) }\end{array}$ & $\begin{array}{l}\text { sulfate (x 1) } \\
\text { S7 } \\
\text { glucuronide (x } \\
\text { 2) } \\
\text { G9, G10 }\end{array}$ & $\begin{array}{l}\text { sulfate (x 1) } \\
\text { S8 } \\
\text { glucuronide (x } \\
\text { 3) } \\
\text { G7, G8, G10 }\end{array}$ & (x 1) & $\begin{array}{l}\text { (x 2) } \\
\text { M10, M12 }\end{array}$ & $\begin{array}{l}\text { (x 2) } \\
\text { M11, M12 }\end{array}$ \\
\hline $\begin{array}{l}\text { dihydroxylated furazadrol } \\
\text { (minor) }\end{array}$ & $\mathrm{N} / \mathrm{D}^{\mathrm{B}}$ & $\mathrm{N} / \mathrm{D}^{\mathrm{B}}$ & $\mathrm{N} / \mathrm{D}^{\mathrm{B}}$ & $(\mathrm{x} 2)$ & $\begin{array}{l}(\mathrm{x} 4) \\
\text { M13-M16 }\end{array}$ & $\begin{array}{l}\text { (x 3) } \\
\text { M13, M15, M16 }\end{array}$ \\
\hline
\end{tabular}

